\title{
A Randomized Controlled Trial on the Efficacy and Safety of Low-Dose hCG in a Short Protocol with GnRH Agonist and Ovarian Stimulation with Recombinant FSH (rFSH) During the Follicular Phase in Infertile Women Undergoing ART
}

\author{
Charalampos Siristatidis $^{1,2}$ (D) $\cdot$ Sofoklis Stavros $^{3}$ (D) Konstantinos Dafopoulos $^{4}$ (D) Theodoros Sergentanis $^{5}$ (D) \\ Ekaterini Domali ${ }^{3}$ (D) $\cdot$ Peter Drakakis $^{3}$ (I) $\cdot$ Dimitrios Loutradis $^{3}$ (I)
}

Received: 27 April 2021 / Accepted: 25 June 2021 / Published online: 12 July 2021

(C) Society for Reproductive Investigation 2021

\begin{abstract}
This study aims to investigate whether the addition of low-dose hCG throughout stimulation in infertile women undergoing IVF improves IVF outcome parameters. This is a prospective, multicenter, randomized, double-blind, placebo-controlled, Phase IIIb clinical study, conducted in three university IVF units. We studied whether the addition of $100 \mathrm{IU}$ hCG/day to a short $\mathrm{GnRH}$ agonist IVF protocol from the onset of the follicular phase (group 1,n=40) or placebo (group 2,n=41) had any impact on the number of high-quality transferred embryos at day 2 and clinical pregnancy rates. The comparison encompassed descriptive statistics, and univariate and multivariate analyses. Concerning the primary outcomes, we found no differences in both the number of high-quality embryos $(\geq 2)$ at day $3[21 / 40(52.5 \%)$ vs. $14 / 41(34.2 \%), p=0.095]$ and clinical pregnancy rates [10/ $40(25 \%)$ vs. $10 / 41(24.4 \%), p=0.949]$, respectively. Similarly, there were no differences concerning the secondary outcomes preset for this trial. According to the results of the multivariate logistic regression analysis, no significant associations were noted for primary outcomes (clinical pregnancy: adjusted $\mathrm{OR}=0.89,95 \%$ CI: $0.29-2.75$; ( $\geq 2$ excellent quality embryos at day 3 : adjusted $\mathrm{OR}=0.54,95 \%$ CI: $0.21-1.42$, with group 1 set as reference category); similarly, no differences were noted with respect to secondary outcomes, except from the increased odds of $\geq 2$ poor-quality embryos at day 3 occurring in group 2 (adjusted OR= 11.69, 95\% CI: 1.29-106.19). The addition of low-dose hCG to a short GnRH agonist protocol for IVF does not improve the number of top-quality embryos and clinical pregnancy rates.
\end{abstract}

Keywords Assisted reproduction $\cdot$ Live birth $\cdot$ hCG $\cdot \mathrm{LH} \cdot$ Embryo quality

\section{Introduction}

The choice of a simple, safe, and cost-effective protocol in controlled ovarian hyperstimulation $(\mathrm{COH})$ is crucial for

Charalampos Siristatidis

harrysiris@gmail.com

1 Assisted Reproduction Unit, Third Department of Obstetrics and Gynecology, "Attikon" Hospital, Medical School, National and Kapodistrian University of Athens, 1 Rimini Str., 12642 Chaidari, Athens, Greece

2 Assisted Reproduction Unit, Second Department of Obstetrics and Gynecology, "Aretaieion" University Hospital, Medical School, National and Kapodistrian University of Athens, 76 Vas. Sofias Av, 11528 Athens, Greece improving the quality of care in assisted reproduction, through high pregnancy rates. The main parameters are the numbers of oocytes retrieved and good-quality embryos available for transfer; both of them are closely related to high live birth rate
3 Assisted Reproduction Unit, First Department of Obstetrics and Gynecology, "Alexandra" Hospital, Medical School, National and Kapodistrian University of Athens, 80 Vas. Sofias Av. and Lourou str., 11528 Athens, Greece

4 Assisted Reproduction Unit, Department of Obstetrics and Gynecology, University Hospital, Faculty of Medicine, School of Health Sciences, University of Thessaly, 41110 Larissa, Greece

5 Department of Clinical Therapeutics, "Alexandra" Hospital, Medical School, National and Kapodistrian University of Athens, 80 Vas. Sofias Av., 11528 Athens, Greece 
[1]. Still, there is an ongoing clinical uncertainty among fertility specialists in the optimal way for treating couples with a low-cost regimen and especially those at a relatively advanced reproductive age.

We know that the increasing luteinizing hormone ( $\mathrm{LH})$ in the follicular phase of the cycle leads to a number of key changes in oocytes and follicular cells, modifying the microand macro-environment of the ovary, and is linked to the normal oocytes' maturation, the ovulation process, and subsequent fertilization and implantation [2-4]. Human chorionic gonadotropin (hCG) has been used as a substitute for $\mathrm{LH}$, due to the high degree of similarity between the $\alpha$-subunits of the two hormones, similarly to FSH, LH, and TSH. Both hCG and LH are heterodimeric glycoproteins with high cysteine content and exert similar activity in inducing ovulation and supporting luteal cells [5]. In contrast, their differences at the molecular level, mainly due to the extra 24 amino acids in the $\beta$-chain, give hCG the ability of possessing additional glycosylation sites and thus a longer half-life, providing a more sustained ovarian stimulation [6], compared to LH: thus, hCG has a higher binding affinity for the receptor [inducing high proapoptotic activity in vitro] and mediates a more potent cAMP increase in human primary granulosa cells $[3,4,7,8]$.

Concerning the supplementation with $\mathrm{r}-\mathrm{LH}$, there is evidence on its positive impact on the outcome parameters in IVF own fresh cycles [9-11], causing a low intrafollicular concentration of vascular endothelial growth factor [12], in women having at least 2 previous failed IVF attempts [13], in poor responders and women over 35 years $[14,15]$, and in oocyte donors [16]. In contrast, there are reports with opposite conclusions $[2,17]$. The current evidence through systematic reviews is rather conflicting: some have failed to confirm a positive impact in the general infertile population $[18,19]$, whereas others report significantly higher clinical pregnancy rates [20], especially in poor responders, in women of advanced reproductive age $[21,22]$, and in those with the previous hypo-response to exogenous FSH stimulation [23].

The rationale for adding daily HCG during $\mathrm{COH}$ in IVF is that, similar to LH, it improves the clinical outcome; notably, the earlier that HCG is added (i.e., at day 1), the more favorable the outcome will be. This starts with an optimal follicular development- $\mathrm{a}$ threshold level of $1.2 \mathrm{IU} / \mathrm{L}$ of LH is necessary to avoid premature luteinization and reduced $\mathrm{COH}$ response $[6,24]$ - due to increased interaction at the $\mathrm{LH} / \mathrm{hCG}$ receptor level [25]. It was reported that higher serum hCG levels are correlated with lower progesterone and a higher number of follicles/oocytes and pregnancy rates [26, 27]. Day 6 hCG concentrations have been shown to predict the frequency of top-quality embryos, ongoing pregnancy, and live birth rates [28]. Concerning dosage, supplementation with hCG from the first day of $\mathrm{COH}$ at a range of $0-150 \mathrm{IU} /$ day was reported as "compatible with good live birth rates" [29]. Similar findings have been reported when hCG is added during the early follicular phase [13, 30-32]. The full mechanism still remains unknown, but hCG seems to enhance the production of a different profile of cytokines and gene expression in both cumulus and endometrial cells, generating a better environment to produce healthier-perhaps fewer-oocytes compared with rLH, higher endometrial receptivity, and implantation potential [28]. Moreover, when added —or replace rFSH - during the late follicular phase, it yields similar results concerning IVF outcomes [33, 34]: the rationale is based on the higher expression of $\mathrm{LH}$ receptors during that phase; thus, $\mathrm{rFSH}$ can be replaced by lower doses of hCG, which are less expensive, and associated with lower incidence of ovarian hyperstimulation syndrome [OHSS]. Similarly, the metaanalysis by Checa et al. supports the use of hCG mainly in the late follicular phase as an efficient alternative to conventional modalities for $\mathrm{COH}$ as it was associated with improved clinical pregnancy rates and reduction in the administered rFSH doses [35].

In this context, we performed a RCT to test the efficacy and safety of adding daily low-dose hCG in a short protocol with $\mathrm{GnRH}$ agonist and ovarian stimulation with $\mathrm{rFSH}$ from the onset of the follicular phase and throughout the duration of stimulation in women of advanced reproductive age undergoing IVF/ICSI.

\section{Materials and Methods}

\section{Study Population}

The present study was designed as a prospective, multicenter, randomized, double-blind, placebo-controlled, two parallel groups of patients, phase IIIb clinical study to deal with the specific research question: to test the efficacy and safety of adding daily low-dose hCG in a short protocol with GnRH agonist and ovarian stimulation with recombinant $\mathrm{FSH}$ $(\mathrm{rFSH})$ from the onset of the follicular phase and throughout the duration of stimulation towards the increase of outcome parameters.

Consent to use anonymous data for research purposes was obtained from all participants, and the research protocol was approved by the Bioethics Committee and the Scientific Council of the Hospital, prior to study initiation (EVD no 47/1/13-3-2017; 4/13-3-17) and the relevant committees (EED 46-17/23-6-2017; EOF IS 44-17/40345/2017). The protocol was registered in the relevant scientific databases (NCT03423527; study name, hCG-GR-001-2016; EudraCT Number 2016-005208-24).

Data was collected on $81 \mathrm{IVF} / \mathrm{ICSI}$ cycles, at the assisted reproduction units of the Third Department of Obstetrics and Gynecology; "Attikon" University Hospital, the First Department of Obstetrics and Gynecology; "Alexandra" University Hospital, Athens; and the Department of 
Obstetrics and Gynecology of University of Thessaly, Greece, and from July 2017 to April 2019. For the eligible population, conclusive data was available on demographics, medical/ reproductive history for both partners. Inclusion criteria were age between 35 and 40 years, physiological menstrual cycles (24-35 days), normal endocrine function (normal PRL and $\mathrm{TSH}, \mathrm{FSH} \leq 15 \mathrm{IU} / \mathrm{ml}$ ), transvaginal ultrasound without pathological findings, free personal medical history, indication for IVF/ICSI [36], and first or second IVF/ICSI cycle. Exclusion criteria included endocrine or metabolic disorders, e.g., PCO (S), pathology of the uterus and/or endometrium, basal FSH levels $>15 \mathrm{IU} / \mathrm{ml}$, surgery in the ovaries, body mass index $(\mathrm{BMI}) \geq 35 \mathrm{~kg} / \mathrm{m} 2$, and age $<35$ years and $>40$ years old.

No other interventions were applied during cycles, apart from the routine protocols described in the regular practice of all IVF Units, which conform to international trends and European standards for appropriate medical management. The GnRH agonist short protocol was used to promote ovarian stimulation, which has been described in one of our previous studies [13]. All procedures, culture conditions and success rates, along with medical and paramedical personnel, were stable throughout the years of the more than 10 years function of all Units, all of which were under standard operating procedures. Embryo transfer took place 3 days after the oocyte retrieval; the maximum of embryos placed was two, according to the Hellenic legislation.

The study group (group 1 [HCG [+] group]) indicated the application of the protocol by adding hCG with the initiation of standard short GnRH agonist protocol for IVF/ICSI with $200 \mathrm{IU}$ rFSH (BEMFOLA, Fertilland Pharma/Gedeon Richter Plc, Hungary), while the control group (group 2 [HCG [-] group]) indicated the application of the same protocol, adding placebo. The addition of hCG (Pregnyl ${ }^{\circledR}$, MSD Greece) at a dose of $100 \mathrm{IU}$ per day was administered subcutaneously and continued until the administration of hCG for follicular maturation/triggering; flacons were prepared centrally and distributed to all involved Units, by one study midwife, using 5000 IU commercial ampoules. As for placebo, an identical flacon with $100 \mathrm{IU}$ containing N/S $0.9 \%$ per day, was injected.

A dynamic database was generated with Microsoft Excel and used for data storage and preprocessing. In total, $62 \mathrm{pa}-$ rameters were available, as categorically reported here: infertility duration and factor, medical and reproductive history, lifestyle information and demographics linked to fertility dynamics, pathology linked to infertility factors for both partners, age at cycle, parity, hormonal profile along with selected information on menstrual cycle characteristics for the female partner and ultrasonographic tests, such as antral follicle count [AFC], and sperm analysis for the male partner. Cycle characteristics of the previous ART cycle and their outcome, together with parameters of ovarian response to ovarian stimulation, were monitored. The full set of parameters is presented in the supplementary file [Supp. file].
Participants were randomly divided into two groups: group 1 and group 2. Randomization was done using sealed opaque envelopes marked as "group 1" and "group 2". The randomization list was prepared centrally for the three participating Units [research centers]. Each patient's assignment to a treatment group was made by calling the Unit's midwife who had 2 envelopes with the above indications and who was not present when the couple was informed by two members of the research team.

\section{Outcome Measures}

The primary outcomes were the number of top-quality transferred embryos and clinical pregnancy rate. Secondary endpoints included the number of follicles [>11,> 14,> $18 \mathrm{~mm}]$ and endometrial thickness on the day of triggering; the total number of retrieved and MII oocytes; the total number of total, top-rated, and frozen embryos; biochemical, ectopic, and multiple pregnancy; miscarriage; and OHSS rates. Definitions of the outcomes were according to the International Glossary on Infertility and Fertility Care [37].

\section{Statistical Analysis and Power Calculation}

As for the sample size calculation, using similar trials as references, we observed that the main differences of outcomes (not very profound in all studies) were achieved in the level of embryos, and especially their quality, leading to higher pregnancy rates $[13,17,29,30]$. Thus, we calculated that samples of 50 participants from each arm would be required, to provide a significance of 0.05 and a power of 0.8 detecting a difference equal to $0.58 *$ standard deviation in the top-quality embryos, including dropouts and missing data. So, the total number of participants required was 90 . Unfortunately, the study was prematurely ended (while relative authorities were notified officially), as one of the main investigators moved to another IVF Unit and mainly because of the Covid-19 pandemic: IVF protocols in all IVF Units worldwide changed from fresh to frozen, so that it was impossible to recruit more patients during the last year.

The statistical analysis encompassed descriptive statistics, univariate, and multivariate analysis. Regarding descriptive statistics, variables were summarized separately by group/ treatment arm; categorical variables were summarized as frequency $(\%)$, whereas median and interquartile range (IQR) were presented for continuous variables. Deviations from normality were evaluated with the Shapiro-Wilk test. For categorical variables, differences between the two treatment arms were evaluated with Pearson's chi-squared test or Fisher's exact test, as appropriate. Regarding continuous variables, differences were assessed with $t$ test for independent samples in case of a normal distribution, whereas the Mann-Whitney- 
Wilcoxon test for independent samples was implemented for variables that were not distributed normally.

Univariate and multivariate logistic regression analysis was performed to evaluate differences in outcomes between the two groups. The outcomes were set as dependent variables; at the multivariate models, the effects of group (group 1 as reference category) were adjusted for age ( $\geq 38$ vs. $<38$ years), BMI ( $\left.\geq 25 \mathrm{vs} .<25 \mathrm{~kg} / \mathrm{m}^{2}\right)$, baseline AMH ( $\left.\geq 1 \mathrm{vs} .<1 \mathrm{ng} / \mathrm{mL}\right)$, and AFC ( $>4$ vs. $\leq 4)$. Odds ratios (ORs) and their $95 \%$ confidence intervals $(95 \% \mathrm{CI})$ were estimated.

Outcomes were categorized a priori as detailed below, to be converted to binary dependent variables in the logistic regression models. Clinical pregnancy (yes vs. no) and excellent quality embryos on day 3 ( $\geq 2$ vs $<2$ ) were the primary outcomes. Concerning secondary outcomes, the $\geq$ median vs $<$ median cutoff was adopted for the number of follicles $>11 \mathrm{~mm},>14 \mathrm{~mm}$, and $>18 \mathrm{~mm}$ at triggering, E2 and P4 at triggering, endometrial thickness at triggering, number of MII oocytes, ET, and frozen embryos; the $\geq 2$ vs $<2$ cutoff was utilized for the number of oocytes, moderate-quality embryos, and poor-quality embryos on day 3 ; the yes vs. no classification was inherently utilized to treat biochemical pregnancy, cycle cancellation, and ovarian hyperstimulation syndrome as dependent variables.

The statistician was blinded to the treatment arm corresponding to each treatment arm/group coding in the dataset. The level of significance was set at 0.05 . Statistical analysis was performed with STATA/SE version 13 statistical software (Stata Corp. College Station, TX, USA).

\section{Results}

An ensemble of 62 parameters and variables was available for $81 \mathrm{IVF} / \mathrm{ICSI}$ cycles, sourcing from a total of 81 infertile couples, with overall successful clinical outcome with clinical pregnancy in $21.6 \%$ of the cycles included.

Descriptive statistics for the two treatment arms are presented in Table 1 . The median age was equal to 38 years in both arms. No significant differences were noted regarding sociodemographic and lifestyle features, including BMI, previous parity, smoking, and alcohol consumption. Unexplained infertility ( $n=16,40 \%$, and $n=13,31.7 \%$ ), as well as male factor infertility ( $n=16,40 \%$, and $n=17,41.5 \%$ ) was the most common underlying cause in both groups. No significant differences were documented in reproductive and clinical history variables, including chronic disease, age at menarche, duration of menstruation, type of infertility, duration of infertility, and the examined cycle being the first IVF/ICSI cycle between the two treatment groups.

Similarly, no significant differences were documented in baseline heart rate, systolic blood pressure, baseline FSH, TSH, LH, E2, AMH, PRL, and AFC, or features of the male partner, including BMI, smoking, alcohol consumption, chronic disease, and proven fertility, between the two treatment arms.

At the univariate analysis examining differences in primary and secondary outcomes between the two groups, no significant differences were noted either for primary or secondary outcomes (Table 2). Specifically, regarding primary outcomes, the clinical pregnancy rate was equal to $25 \%(10 / 40)$ and $24.4 \%(10 / 41), p=0.949$, whereas the proportion of two or more excellent quality embryos on day 3 was $52.5 \%(21 / 40)$ and $34.2 \%(14 / 41)$ in groups 1 and 2, respectively, $p=0.095$.

The results of the multivariate logistic regression analysis comparing the odds of the primary and secondary outcomes between the two groups are detailed in Table 3. No significant associations were noted for primary outcomes (clinical pregnancy: adjusted OR=0.89, 95\% CI: $0.29-2.75$; two or more excellent quality embryos on day 3 : adjusted $\mathrm{OR}=0.54,95 \%$ CI: $0.21-1.42$, with group 1 set as reference category). With respect to secondary outcomes, no differences were noted, except for increased odds of two or more poor-quality embryos on day 3 occurring in group 2 (adjusted $\mathrm{OR}=11.69$, 95\% CI: 1.29-106.19).

\section{Discussion}

We performed a prospective, multicenter, randomized, phase IIIb clinical study to test the efficacy and safety of adding $100 \mathrm{IU}$ hCG/day in a short IVF protocol with GnRH agonist and rFSH from the onset of the follicular phase and throughout the duration of stimulation. The two groups demonstrated similar demographic baseline characteristics. The analysis showed similar rates of the number of top quality at day 3 and clinical pregnancy in the two groups studied. The same results were observed in the secondary endpoints of this study, including numbers of total, top-rated, and frozen embryos; biochemical, ectopic, and multiple pregnancy; miscarriage; and OHSS rates.

The rationale of daily hCG supplementation is based on its positive effects on the follicular microenvironment, endometrial receptivity, and the immune system response during $\mathrm{COH}$ [38]. Notably, this effect is dose dependent, as larger doses could result in an androgenic environment; a daily dose of 100-150 IU was proposed as optimal [29, 39], constituting the testing dose in our trial.

The comparison with the current literature has resulted in conflicting results.

On one hand, high serum hCG levels along with the addition of hCG to $\mathrm{rFSH}$ have been correlated with a high number of follicles/oocytes and pregnancy rates, irrespective of the $\mathrm{COH}$ protocol used $[26,27,40]$. In a retrospective study, the addition of hCG led to favorable IVF outcomes in women above 40 years of age [41]. Similarly, in a dose-response 
Table 1 Descriptive statistics in the two study arms

\begin{tabular}{|c|c|c|c|}
\hline & Group $1(n=40)$ & Group $2(n=41)$ & $p$ \\
\hline \multicolumn{4}{|l|}{ Sociodemographic and lifestyle features } \\
\hline Age, years, median [IQR] & $38[4]$ & $38[3]$ & $0.980^{\mathrm{T}}$ \\
\hline BMI, $\mathrm{kg} / \mathrm{m}^{2}$, median [IQR] & $22.6[4.6]$ & $23.9[6.1]$ & $0.292^{\mathrm{M}}$ \\
\hline Previous parity, $n(\%)$ & $3(7.5)$ & $4(9.8)$ & $0.718^{\mathrm{C}}$ \\
\hline Smoking, $>4$ cigarettes/day, $n(\%)$ & $12(30.0)$ & $16(39.0)$ & $0.393^{\mathrm{C}}$ \\
\hline Alcohol consumption, $>4$ cups/week, $n(\%)$ & $0(0.0)$ & $1(2.4)$ & $>0.999^{\mathrm{F}}$ \\
\hline \multicolumn{4}{|l|}{ Reproductive and clinical history } \\
\hline Chronic disease, $n(\%)$ & $14(35.0)$ & $12(29.3)$ & $0.581^{\mathrm{C}}$ \\
\hline Age at menarche, years, median [IQR] & $13.0[1.5]$ & $13.0[1.0]$ & $0.313^{\mathrm{T}}$ \\
\hline Duration of menstruation, days, median [IQR] & $5[0]$ & $5[1.5]$ & $0.906^{\mathrm{T}}$ \\
\hline Type of infertility, $n(\%)$ & & & $0.887^{\mathrm{F}}$ \\
\hline Tubal & $4(10.0)$ & $4(9.8)$ & \\
\hline Male & $16(40.0)$ & $17(41.5)$ & \\
\hline Anovulatory & $0(0.0)$ & $1(2.4)$ & \\
\hline Male and tubal & $4(10.0)$ & $6(14.6)$ & \\
\hline Unexplained & $16(40.0)$ & $13(31.7)$ & \\
\hline First IVF/ICSI cycle, $n(\%)$ & $26(65.0)$ & $33(80.5)$ & $0.117^{\mathrm{C}}$ \\
\hline \multicolumn{4}{|l|}{ Findings at baseline } \\
\hline Heart rate, bpm, median [IQR] & $75[10]$ & $77[9]$ & $0.217^{\mathrm{M}}$ \\
\hline Systolic blood pressure, $\mathrm{mmHg}$, median [IQR] & $116[11]$ & $115[11]$ & $0.892^{\mathrm{T}}$ \\
\hline Duration of infertility, years, median [IQR] & $3.5[2.0]$ & $3.0[4.0]$ & $0.977^{\mathrm{M}}$ \\
\hline \multicolumn{4}{|l|}{ Baseline hormonal measurements } \\
\hline FSH, (mIU/ml), median [IQR] & $7.4[5.1]$ & $8.0[3.4]$ & $0.882^{\mathrm{T}}$ \\
\hline TSH, (mIU/lt), median [IQR] & $1.7[1.0]$ & $1.7[0.9]$ & $0.627^{\mathrm{T}}$ \\
\hline $\mathrm{LH},(\mathrm{mIU} / \mathrm{ml})$, median $[\mathrm{IQR}]$ & $5.7[4.3]$ & $6.1[3.5]$ & $0.685^{\mathrm{M}}$ \\
\hline E2, pg/ml, median [IQR] & $39.5[16.2]$ & $43.3[21.3]$ & $0.806^{\mathrm{M}}$ \\
\hline $\mathrm{AMH}, \mathrm{ng} / \mathrm{mL}$, median $[\mathrm{IQR}]$ & $1.4[1.5]$ & $1.0[1.3]$ & $0.106^{\mathrm{M}}$ \\
\hline PRL, ng/mL, median [IQR] & $14.9[10.5]$ & $13.0[10.3]$ & $0.887^{\mathrm{M}}$ \\
\hline AFC, median [IQR] & $8.5[4.0]$ & $7.0[4.0]$ & $0.518^{\mathrm{M}}$ \\
\hline \multicolumn{4}{|l|}{ Features of the male partner } \\
\hline BMI, kg/m², median [IQR] & $27.5[6.5]$ & $27.6[4.7]$ & $0.702^{\mathrm{M}}$ \\
\hline Smoking, $>4$ cigarettes/day, $n(\%)$ & $20(50.0)$ & $23(56.1)$ & $0.582^{\mathrm{C}}$ \\
\hline Alcohol consumption, $>4$ cups/week, $n(\%)$ & $1(2.5)$ & $2(4.9)$ & $>0.999^{\mathrm{F}}$ \\
\hline Chronic disease, $n(\%)$ & $10(25.0)$ & $7(17.1)$ & $0.381^{\mathrm{C}}$ \\
\hline Proven fertility, $n(\%)$ & $10(25.0)$ & $15(36.6)$ & $0.259^{\mathrm{C}}$ \\
\hline
\end{tabular}

${ }^{\mathrm{C}} p$ value derived from Pearson's chi-squared test; ${ }^{\mathrm{F}} p$ value derived from Fisher's exact test; ${ }^{\mathrm{M}} p$ value derived from Mann-Whitney-Wilcoxon test for independent samples; ${ }^{\mathrm{T}} p$ value derived from t-test; $A F C$, antral follicle count; $A M H$, anti-Müllerian hormone; $B M I$, body mass index; $E 2$, estradiol; $F S H$, follicle-stimulating hormone; $I Q R$, interquartile range; $L H$, luteinizing hormone; $P R L$, prolactin; $T S H$, thyroid-stimulating hormone
RCT, increasing doses of hCG supplementation stimulated the intrafollicular concentration of both estradiol and androgens, with a shift toward a more androgenic milieu which was apparent in large follicles with oocytes giving rise to goodquality embryos [39].

In contrast, our study did not reveal any statistically significant difference and thus clinical benefit through the addition of daily hCG, in terms of the number of top-quality embryos and clinical pregnancy. Notably, the difference demonstrated in the number of top-quality embryos available for transfer and the poor-quality embryos at day 3 , between the two groups $(52.5 \%$ vs $34.2 \%$ and $2.5 \%$ vs $17.7 \%$, respectively), did not manage to reach statistical significance. A different, compared to our study, practice of $\mathrm{hCG}$ administration has been investigated in a Cochrane review (33); authors aimed to assess the effectiveness and safety of low-dose hCG when replacing FSH for $\mathrm{COH}$ : that did not reduce the chance of ongoing and clinical pregnancy and resulted in an equivalent 
Table 2 Primary and secondary outcomes in the two study arms

\begin{tabular}{|c|c|c|c|}
\hline & Group $1(n=40)$ & Group $2(n=41)$ & $p$ \\
\hline \multicolumn{4}{|l|}{ Primary outcomes } \\
\hline Clinical pregnancy, $n(\%)$ & $10(25.0)$ & $10(24.4)$ & $0.949^{\mathrm{C}}$ \\
\hline Excellent quality embryos on day $3(\geq 2), \mathrm{n}(\%)$ & $21(52.5)$ & $14(34.2)$ & $0.095^{\mathrm{C}}$ \\
\hline \multicolumn{4}{|l|}{ Secondary outcomes } \\
\hline Number of follicles $>11 \mathrm{~mm}$ at triggering, median [IQR] & $6[6]$ & $6[5]$ & $0.996^{\mathrm{M}}$ \\
\hline Number of follicles $>14 \mathrm{~mm}$ at triggering, median [IQR] & $5[5]$ & $6[4]$ & $0.377^{\mathrm{M}}$ \\
\hline Number of follicles $>18 \mathrm{~mm}$ at triggering, median [IQR] & $3[4]$ & $3[4]$ & $0.568^{\mathrm{M}}$ \\
\hline E2 at triggering, $\mathrm{pg} / \mathrm{ml}$, median $[\mathrm{IQR}]$ & 1770 [1742] & 1100 [1182] & $0.144^{\mathrm{M}}$ \\
\hline $\mathrm{P} 4$ at triggering, $\mathrm{ng} / \mathrm{ml}$, median [IQR] & $1.1[1.1]$ & $0.9[0.8]$ & $0.116^{\mathrm{M}}$ \\
\hline Endometrial thickness at triggering, mm, median [IQR] & $9.0[2.0]$ & $9.0[2.2]$ & $0.917^{\mathrm{M}}$ \\
\hline Number of oocytes $(\geq 2), n(\%)$ & $32(80.0)$ & $34(82.9)$ & $0.735^{\mathrm{C}}$ \\
\hline Number of MII oocytes, median [IQR] & $3[5]$ & $3[2]$ & $0.735^{\mathrm{M}}$ \\
\hline Moderate quality embryos on day $3(\geq 2), n(\%)$ & $5(12.5)$ & $7(17.1)$ & $0.562^{\mathrm{C}}$ \\
\hline Poor-quality embryos on day $3(\geq 2), n(\%)$ & $1(2.5)$ & $7(17.1)$ & $0.057^{\mathrm{F}}$ \\
\hline ET embryos, median [IQR] & $2[1]$ & $2[1]$ & $0.545^{\mathrm{M}}$ \\
\hline Frozen embryos, median [IQR] & $0[3]$ & $0[1]$ & $0.894^{\mathrm{M}}$ \\
\hline Biochemical pregnancy, $n(\%)$ & $11(27.5)$ & $12(29.3)$ & $0.860^{\mathrm{C}}$ \\
\hline Cycle cancellation, $n(\%)$ & $8(20.0)$ & $7(17.1)$ & $0.735^{\mathrm{C}}$ \\
\hline Spontaneous abortion, $n(\%)^{*}$ & $1(3.1)$ & $0(0.0)$ & $0.485^{\mathrm{F}}$ \\
\hline Ovarian hyperstimulation syndrome, $n(\%)$ & $3(7.5)$ & $1(2.4)$ & $0.359^{\mathrm{F}}$ \\
\hline
\end{tabular}

*regarding spontaneous abortion, the denominator was the number of women on whom cycles were not cancelled (32 and 34 in groups 1 and 2 , respectively); ${ }^{\mathrm{C}} p$ value derived from Pearson's chi-squared test; ${ }^{\mathrm{F}} p$ value derived from Fisher's exact test; ${ }^{\mathrm{M}} p$ value derived from Mann-WhitneyWilcoxon test for independent samples; E2, estradiol; ET, embryo transfer; IQR, interquartile range; P4, progesterone

number of oocytes retrieved, while the consumption of FSH was lower. In a similar study carried out later [42], there were no significant differences between pregnancy rates in the two study groups. In a recent RCT, the administration of low-dose hCG daily from the early follicular phase in PCOS patients through a progesterone protocol did not manage to improve the number of retrieved and mature oocytes and good-quality embryos, along with implantation and clinical pregnancy rates [43].

Concerning the secondary outcomes preset for this RCT, we found no significant differences between the two groups. For example, the level of E2 at triggering was higher in the hCG group (1770 vs. $1100 \mathrm{pg} / \mathrm{ml}$ ), indicating better cytoplasmic maturation and probably better expected quality of oocytes/embryos [44], but not significantly. Similar results have been reported in fertilization, implantation, and ongoing pregnancy rates $[40,41]$ and when HCG was administered in the late follicular phase [45]. Finally, we observed a nonsignificant increase of the incidence of OHSS in the hCG group ( $7.5 \%$ vs. $2.4 \%$ ); all cases concerned mild/moderate OHSS not requiring hospitalization; all of them were canceled, as no HCG triggering was applied, except from one case that ended in clinical pregnancy.
Importantly, two meta-analyses ended up with conflicting conclusions $[34,35]$ : reasons for that were: the significant diversity of the inclusion and exclusion criteria of the included studies, the heterogeneity of the reported outcomes, and the methodological assessment of the outcomes leading to different interpretations of the results.

Among the strengths of our trial, we have to point out the properly taken steps from the conception of the protocol up to the publication of the results, in an effort to minimize all domains of biases. Limitations include the small cohort size, which was based on the power calculation on the number of top-quality embryos at day 3 and not on live birth. Others include the use of the specific $\mathrm{COH}$ protocol and the small dose of hCG (100 IU/day) used.

The co-administration of daily hCG during $\mathrm{COH}$ for IVF seems a promising alternative to conventional modalities, mainly related to the enhancement of LH activity. The results of this RCT showed that the addition of $100 \mathrm{IU}$ of hCG/day from the start of stimulation with rFSH in a GnRH agonist short protocol does not seem to increase significantly the rate of top-quality embryos and clinical outcomes. As yet, the relevant data in the literature is conflicting, necessitating more studies to 
Table 3 Univariate and multivariate logistic regression analysis examining outcomes in the two study arms. Group 1 was set as the reference category

Outcome compared categories $\quad$ Crude OR (95\%CI) Adjusted OR (95\% CI)*

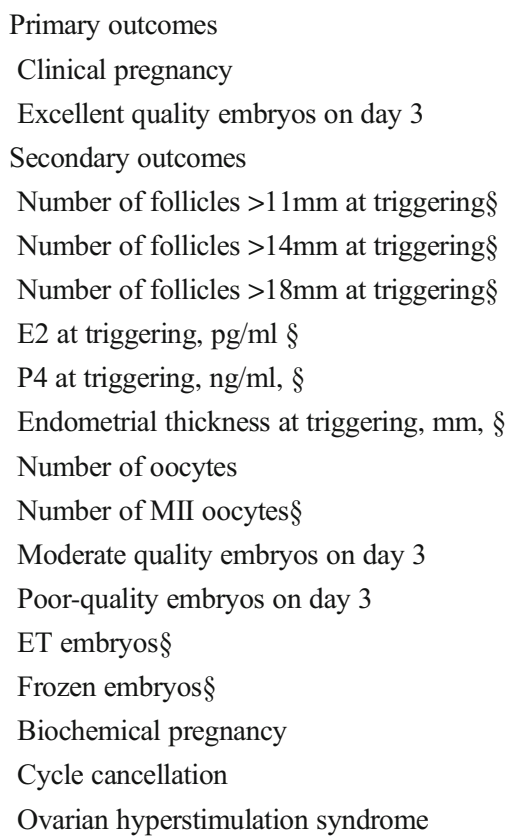

$\begin{array}{ll}\text { Yes vs. no } & 0.97(0.35-2.66) \\ \geq 2 \text { vs. }<2 & 0.47(0.19-1.15) \\ \geq 6 \text { vs. }<6 & \\ \geq 6 \text { vs. }<6 & 1.28(0.52-3.13) \\ \geq 3 \text { vs. }<3 & 1.73(0.72-4.16) \\ \geq 1300 \text { vs }<1300 & 1.16(0.48-2.78) \\ \geq 0.95 \text { vs. }<0.95 & 0.47(0.19-1.15) \\ \geq 9 \text { vs. }<9 & 0.60(0.25-1.46) \\ \geq 2 \text { vs. }<2 & 0.94(0.39-2.28) \\ \geq 3 \text { vs. }<3 & 1.21(0.39-3.73) \\ \geq 2 \text { vs. }<2 & 0.95(0.40-2.27) \\ \geq 2 \text { vs. }<2 & 1.44(0.42-4.98) \\ \geq 2 \text { vs. }<2 & 8.03(0.94-68.60) \\ \geq 1 \text { vs. } 0 & 0.75(0.30-1.87) \\ \text { Yes vs. no } & 1.20(0.48-3.00) \\ \text { Yes vs. no } & 1.09(0.41-2.87) \\ \text { Yes vs. no } & 0.82(0.27-2.53)\end{array}$

$0.89(0.29-2.75)$

$0.54(0.21-1.42)$

$1.50(0.54-4.19)$

$2.38(0.86-6.60)$

$1.30(0.51-3.32)$

$0.60(0.24-1.56)$

$0.63(0.24-1.64)$

$0.94(0.37-2.41)$

$1.59(0.47-5.43)$

$1.27(0.48-3.34)$

$1.69(0.44-6.49)$

$11.69(1.29-106.19)$

$0.84(0.32-2.20)$

$1.51(0.53-4.30)$

$1.05(0.36-3.04)$

$0.81(0.25-2.66)$

$0.45(0.04-4.79)$

*adjusted for age ( $\geq 38$ vs. $<38$ years), BMI $\left(\geq 25\right.$ vs. $<25 \mathrm{~kg} / \mathrm{m}^{2}$ ), baseline AMH ( $\geq 1$ vs. $<1 \mathrm{ng} / \mathrm{mL}$ ), and AFC ( $>4$ vs. $\left.\leq 4\right)$; $\S$ the median was set as the cutoff values for these outcomes. No analysis on spontaneous abortion was presented, as only one event was noted

clarify the role, pathways of action, and the effectiveness of hCG and other synthetic gonadotropins - with individual isoform composition and receptor binding properties-in $\mathrm{COH}$ for IVF/ICSI. Also, other infertile populations, womens' age groups, protocols, and doses should also be tested to prove or disprove the value of daily hCG administration.

Supplementary Information The online version contains supplementary material available at https://doi.org/10.1007/s43032-021-00683-3.

Acknowledgements The authors wish to thank the medical, paramedical, and laboratory teams of the assisted reproduction units of all University hospitals in Greece for the collection of data, especially the trainees of the Third Department of Obstetrics and Gynecology, Attikon Hospital and Drs Panagiotis Krokos, Athanasia Despotidi and Athanasia Gkamprana and, from the First Department of Obstetrics and Gynecology, Alexandra Hospital, Dr Alexandros Psarris and senior nurse Ritsa Bletsa.

Author contribution Charalampos Siristatidis and Dimitrios Loutradis are responsible for the study conception and design. All authors contributed to patients' recruitment and selection, material preparation, and data collection. Statistical analysis was performed by Theodoros Sergentanis. The first draft of the manuscript was written by Charalampos Siristatidis and improved by all authors. All authors commented on previous versions of the manuscript. All authors read and approved the final manuscript.

Availability of data and material The data can be shared upon request from the editorial team of the journal.

\section{Declarations}

Ethics approval All procedures performed were in accordance with the ethical standards of the scientific council and by the bioethics committee and the scientific council of the hospital, prior to study initiation (EIRBM no 47/1/13-3-2017; 4/13-3-17) and the relevant committees, National Ethics Committee and National Organization for Medicines, respectively (EED 46-17/23-6-2017; EOF IS 44-17/40345/2017), and with the 1964 Helsinki Declaration and its later amendments or comparable ethical standards. The protocol was registered in the relevant databases (NCT03423527; study name: hCG-GR-001-2016; EudraCT Number 2016-005208-24).

Consent to participate All authors have given their consent for participation and publication of the study. All patients have signed the appropriate forms, for their inclusion in the study.

Conflict of interest The authors declare no competing interests. 


\section{References}

1. Sunkara SK, Rittenberg V, Raine-Fenning N, Bhattacharya S, Zamora J, Coomarasamy A. Association between the number of eggs and live birth in IVF treatment: an analysis of 400135 treatment cycles. Hum Reprod. 2011;26(7):1768-74. https://doi.org/10. 1093/humrep/der106.

2. Balasch J, Creus M, Fábregues F, Civico S, Carmona F, Puerto B, et al. The effect of exogenous luteinizing hormone $[\mathrm{LH}]$ on oocyte viability: evidence from a comparative study using recombinant human follicle-stimulating hormone $[\mathrm{FSH}]$ alone or in combination with recombinant LH for ovarian stimulation in pituitarysuppressed women undergoing assisted reproduction. J Assist Reprod Genet. 2001;18(5):250-6. https://doi.org/10.1023/a: 1016662100572

3. Casarini L, Lispi M, Longobardi S, Milosa F, La Marca A, Tagliasacchi D, et al. LH and hCG action on the same receptor results in quantitatively and qualitatively different intracellular signalling. PLoS One. 2012;7(10):e46682. https://doi.org/10.1371/ journal.pone. 0046682

4. Casarini L, Riccetti L, De Pascali F, Nicoli A, Tagliavini S, Trenti $\mathrm{T}$, et al. Follicle-stimulating hormone potentiates the steroidogenic activity of chorionic gonadotropin and the anti-apoptotic activity of luteinizing hormone in human granulosa-lutein cells in vitro. Mol Cell Endocrinol. 2016;422:103-14. https://doi.org/10.1016/j.mce. 2015.12.008.

5. Chandrasekher YA, Hutchison JS, Zelinski-Wooten MB, Hess DL, Wolf DP, Stouffer RL. Initiation of periovulatory events in primate follicles using recombinant and native human luteinizing hormone to mimic the midcycle gonadotropin surge. J Clin Endocrinol Metab. 1994;79(1):298-306. https://doi.org/10.1210/jcem.79.1. 8027245 .

6. Ezcurra D, Humaidan P. A review of luteinising hormone and human chorionic gonadotropin when used in assisted reproductive technology. Reprod Biol Endocrinol. 2014;12:95. https://doi.org/ 10.1186/1477-7827-12-95.

7. Casarini L, La Marca A, Lispi M, Longobardi S, Pignatti E, Simoni M. Proceedings of the 27th annual meeting of the European society of human reproduction \& embryology: 3-6 July 2011; Stockholm, Sweden. Non-equivalence of LH and hCG: an in vitro study. 2011; p. 312

8. Dinopoulou V, Drakakis P, Kefala S, Kiapekou E, Bletsa R, Anagnostou E, et al. Effect of recombinant-LH and hCG in the absence of FSH on in vitro maturation [IVM] fertilization and early embryonic development of mouse germinal vesicle [GV]-stage oocytes. Reprod Biol. 2016 Jun;16(2):138-46. https://doi.org/10. 1016/j.repbio.2016.01.004.

9. Franco JG Jr, Baruffi RL, Oliveira JB, Mauri AL, Petersen CG, Contart P, et al. Effects of recombinant LH supplementation to recombinant FSH during induced ovarian stimulation in the GnRH-agonist protocol: a matched case-control study. Reprod Biol Endocrinol. 2009;7:58. https://doi.org/10.1186/1477-7827-758.

10. Paterson ND, Foong SC, Greene CA. Improved pregnancy rates with luteinizing hormone supplementation in patients undergoing ovarian stimulation for IVF. J Assist Reprod Genet. 2012;29:57983. https://doi.org/10.1007/s10815-012-9740-z.

11. Drakakis P, Loutradis D, Kallianidis K, Liapi A, Milingos S, Makrigiannakis A, et al. Small doses of LH activity are needed early in ovarian stimulation for better quality oocytes in IVF-ET. Eur J Obstet Gynecol Reprod Biol. 2005;121(1):77-80. https://doi. org/10.1016/j.ejogrb.2004.12.021.

12. Pezzuto A, Ferrari B, Coppola F, Nardelli GB. LH supplementation in down-regulated women undergoing assisted reproduction with baseline low serum LH levels. Gynecol Endocrinol. 2009;26:11824. https://doi.org/10.3109/09513590903215516.

13. Drakakis P, Loutradis D, Beloukas A, Sypsa V, Anastasiadou V, Kalofolias G, et al. Early hCG addition to rFSH for ovarian stimulation in IVF provides better results and the cDNA copies of the hCG receptor may be an indicator of successful stimulation. Reprod Biol Endocrinol. 2009; 7:110. https://doi.org/10.1186/1477-7827-7110.

14. Rongieres C, Poirier V, Celebi C, Pirrello O, Bettahar K, Lehert P. Adding luteinizing hormone to follicle stimulating hormone during ovarian stimulation increases pregnancy rate in IVF for poor ovarian responders. Fertil Steril. 2012;98:S78. https://doi.org/10.1016/j. fertnstert.2012.07.281.

15. Mignini Renzini M, Brigante C, Coticchio G, Dal Canto M, Caliari I, Comi R, et al. Retrospective analysis of treatments with recombinant FSH and recombinant LH versus human menopausal gonadotropin in women with reduced ovarian reserve. J Assist Reprod Genet. 2017;34(12):1645-51. https://doi.org/10.1007/s10815-0171034-z.

16. Acevedo B, Sanchez M, Gomez JL, Cuadros J, Ricciarelli E, Hernandez ER. Luteinizing hormone supplementation increases pregnancy rates in gonadotropin-releasing hormone antagonist donor cycles. Fertil Steril. 2004;82:343-7. https://doi.org/10.1016/j. fertnstert.2004.03.020.

17. Marrs R, Meldrum D, Muasher S, Schoolcraft W, Werlin L, Kelly E. Randomized trial to compare the effect of recombinant human FSH [follitropin alfa] with or without recombinant human LH in women undergoing assisted reproduction treatment. Reprod BioMed Online. 2004;8(2):175-82. https://doi.org/10.1016/s14726483[10]60513-5.

18. Kolibianakis EM, Kalogeropoulou L, Griesinger G, Papanikolaou EG, Papadimas J, Bontis J, et al. Among patients treated with FSH and $\mathrm{GnRH}$ analogues for in vitro fertilization, is the addition of recombinant LH associated with the probability of live birth? A systematic review and meta-analysis. Hum Reprod Update. 2007;13:445-52. https://doi.org/10.1093/humupd/dmm008.

19. Mochtar MH, Danhof NA, Ayeleke RO, Van der Veen F, van Wely $\mathrm{M}$. Recombinant luteinizing hormone $[\mathrm{rLH}]$ and recombinant follicle stimulating hormone $[\mathrm{rFSH}]$ for ovarian stimulation in IVF/ICSI cycles. Cochrane Database Syst Rev. 2017;(5) Art. No.: CD005070. https://doi.org/10.1002/14651858.CD005070.pub3.

20. Hill MJ, Levens ED, Levy G, Ryan ME, Csokmay JM, DeCherney $\mathrm{AH}$, et al. The use of recombinant luteinizing hormone in patients undergoing assisted reproductive techniques with advanced reproductive age: a systematic review and meta-analysis. Fertil Steril. 2012;97:1108-14. https://doi.org/10.1016/j.fertnstert.2012.01.130.

21. Hill MJ, Levy G, Levens ED. Does exogenous LH in ovarian stimulation improves assisted reproduction success? An appraisal of the literature. Reprod BioMed Online. 2012;24:261-71. https://doi.org/ 10.1016/j.rbmo.2011.12.005.

22. Lehert P, Kolibianakis EM, Venetis CA, Schertz J, Saunders H, Arriagada $\mathrm{P}$, et al. Recombinant human follicle-stimulating hormone $[\mathrm{r}-\mathrm{hFSH}]$ plus recombinant luteinizing hormone versus $\mathrm{r}$ $\mathrm{hFSH}$ alone for ovarian stimulation during assisted reproductive technology: systematic review and meta-analysis. Reprod Biol Endocrinol. 2014;12:17. https://doi.org/10.1186/1477-7827-12-17.

23. Conforti A, Esteves SC, Di Rella F, Strina I, De Rosa P, Fiorenza A, et al. The role of recombinant $\mathrm{LH}$ in women with hypo-response to controlled ovarian stimulation: a systematic review and meta-analysis. Reprod Biol Endocrinol. 2019;17(1):18. https://doi.org/10. 1186/s12958-019-0460-4.

24. Rodgers M, Mitchell R, Lambert A, Peers N, Robertson WR. Human chorionic gonadotrophin contributes to the bioactivity of Pergonal. Clin Endocrinol. 1992;37(6):558-64. https://doi.org/10. 1111/j.1365-2265.1992.tb01488.x. 
25. JCEM. Recombinant human luteinizing hormone [LH] to support recombinant human follicle-stimulating hormone $[\mathrm{FSH}]$-induced follicular development in LH- and FSH-deficient anovulatory women: a dose-finding study. The European Recombinant Human LH Study Group. J Clin Endocrinol Metab. 1998;83(5): 1507-14.

26. Andersen AN, Devroey P, Arce JC. Clinical outcome following stimulation with highly purified $\mathrm{hMG}$ or recombinant FSH in patients undergoing IVF: a randomized assessor-blind controlled trial. Hum Reprod. 2006;21(12):3217-27. https://doi.org/10.1093/ humrep/del284.

27. Andersen AN. Lack of association between endogenous LH and pregnancy in $\mathrm{GnRH}$ antagonist protocols. Reprod BioMed Online. 2011;23:692-4. https://doi.org/10.1016/j.rbmo.2011.08.009.

28. Smitz J, Andersen AN, Devroey P, Arce JC. Endocrine profile in serum and follicular fluid differs after ovarian stimulation with HPhMG or recombinant FSH in IVF patients. Hum Reprod. 2007;22: 676-87. https://doi.org/10.1093/humrep/del445.

29. Thuesen LL, Loft A, Egeberg AN, Smitz J, Petersen JH, Andersen AN. A randomized controlled dose-response pilot study of addition of hCG to recombinant FSH during controlled ovarian stimulation for in vitro fertilization. Hum Reprod. 2012;27(10):3074-84. https://oi.org/10.1093/humrep/des256.

30. Beretsos P, Partsinevelos GA, Arabatzi E, Drakakis P, Mavrogianni $\mathrm{D}$, Anagnostou $\mathrm{E}$, et al. "hCG priming" effect in controlled ovarian stimulation through a long protocol. Reprod Biol Endocrinol. 2009;7:91. https://doi.org/10.1186/1477-7827-7-91.

31. Partsinevelos GA, Antonakopoulos N, Kallianidis K, Drakakis P, Anagnostou E, Bletsa R, et al. Addition of low-dose hCG to rFSH during ovarian stimulation for IVF/ICSI: is it beneficial? Clin Exp Obstet Gynecol. 2016;43(6):818-25.

32. Blockeel C, De Vos M, Verpoest W, Stoop D, Haentjens P, Devroey P. Can 200 IU of hCG replace recombinant FSH in the late follicular phase in a GnRH-antagonist cycle? A pilot study. Hum Reprod. 2009;24(11):2910-6. https://doi.org/10.1093/ humrep/dep253.

33. Martins WP, Vieira AD, Figueiredo JB, Nastri CO. FSH replaced by low-dose hCG in the late follicular phase versus continued FSH for assisted reproductive techniques. Cochrane Database Syst Rev. 2013;(3):CD010042. https://doi.org/10.1002/14651858. CD010042.pub2.

34. Santi D, Casarini L, Alviggi C, Simoni M. Efficacy of folliclestimulating hormone $[\mathrm{FSH}]$ alone, $\mathrm{FSH}+$ luteinizing hormone, human menopausal gonadotropin or FSH + human chorionic gonadotropin on assisted reproductive technology outcomes in the "personalized" medicine era: a meta-analysis. Front Endocrinol (Lausanne). 2017;8:114. https://doi.org/10.3389/fendo.2017. 00114.

35. Checa MA, Espinós JJ, Requena A. Efficacy and safety of human chorionic gonadotropin for follicular phase stimulation in assisted reproduction: a systematic review and meta-analysis. Fertil Steril. 2012;97(6):1343-50.

36. NICE [National Institute for Health and Care Excellence]; Fertility problems: assessment and treatment. www.nice.org.uk/guidance/ CG156.2017. Assessed 20 Jul 2020.

37. Zegers-Hochschild F, Adamson GD, Dyer S, Racowsky C, de Mouzon J, Sokol R, et al. The international glossary on infertility and fertility care, 2017. Hum Reprod. 2017;32(9):1786-801. https://doi.org/10.1093/humrep/dex234.

38. Theofanakis C, Athanasiou V, Liokari E, Stavrou S, Sakellariou M, Athanassiou AI, et al. The impact of HCG in IVF treatment: does it depend on age or on protocol? J Gynecol Obstet Hum Reprod. 2019;48(5):341-5. https://doi.org/10.1016/j.jogoh.2019.02.012.

39. Thuesen LL, Andersen AN, Loft A, Smitz J. Intrafollicular endocrine milieu after addition of hCG to recombinant FSH during controlled ovarian stimulation for in vitro fertilization. J Clin Endocrinol Metab. 2014;99(2):517-26. https://doi.org/10.1210/jc. 2013-1528.

40. Andersen CY, Elbaek HO, Alsbjerg B, Laursen RJ, Povlsen BB, Thomsen L, et al. Daily low-dose hCG stimulation during the luteal phase combined with GnRHa triggered IVF cycles without exogenous progesterone: a proof of concept trial. Hum Reprod. 2015;30(10):2387-95. https://doi.org/10.1093/humrep/dev184.

41. Gomaa H, Casper RF, Esfandiari N, Chang P, Bentov Y. Addition of low dose hCG to rFSh benefits older women during ovarian stimulation for IVF. Reprod Biol Endocrinol. 2012;10:55. https:// doi.org/10.1186/1477-7827-10-55.

42. Aghahosseini M, Aleyasin A, Yekehtaz H, Kashani L. Low-dose human chorionic gonadotropin adjunct to an antagonist protocol in assisted reproductive technology: a randomized trial study. Acta Med Iran. 2014;52(6):430-7.

43. Zhu X, Fu Y. Randomized, controlled pilot study of Low-dose human chorionic gonadotropin administration beginning from the early follicular phase for women with polycystic ovarian syndrome undergoing ovarian stimulation using the progesterone protocol. Front Endocrinol (Lausanne). 2019;10:875. https://doi.org/10. 3389/fendo.2019.00875.

44. Loutradis D, Drakakis P, Kallianidis K, Milingos S, Dendrinos S, Michalas S. Oocyte morphology correlates with embryo quality and pregnancy rate after intracytoplasmic sperm injection. Fertil Steril. 1999;72(2):240-4. https://doi.org/10.1016/s0015-0282[99]002332.

45. Serafini P, Yadid I, Motta EL, Alegretti JR, Fioravanti J, Coslovsky M. Ovarian stimulation with daily late follicular phase administration of low-dose human chorionic gonadotropin for in vitro fertilization: a prospective, randomized trial. Fertil Steril. 2006;86(4): 830-8. https://doi.org/10.1016/j.fertnstert.2006.02.110.

Publisher's Note Springer Nature remains neutral with regard to jurisdictional claims in published maps and institutional affiliations. 